\title{
Management of Acute Kidney Injury in the Setting of Acute Respiratory Distress Syndrome: Review Focusing on Ventilation and Fluid Management Strategies
}

\author{
Vandan D. Upadhyaya ${ }^{\mathrm{a}}$, Mohammed Z. Shariffa, Roy O. Mathew ${ }^{\mathrm{b}}$, \\ Mohammad A. Hossain ${ }^{a}$, Arif Asifa, d, Tushar J. Vachharajanic
}

\begin{abstract}
Acute respiratory distress syndrome (ARDS) is a major cause of mortality in adults with acute hypoxic respiratory failure and can predispose those afflicted to develop acute kidney injury (AKI). In the setting where AKI and ARDS overlap, incidence of mortality, length of intensive care unit stay, and complexity of management increases drastically. Lung protective ventilation strategy and conservative fluid management are the main focus of therapy in patients with ARDS, but have major implications on renal function. This review aims to provide concise discussion of pathophysiology, ventilation, and fluid management strategies as it relates to AKI in the setting of ARDS.
\end{abstract}

Keywords: Acute respiratory distress; Acute kidney injury; Acute lung injury; Ventilation strategy

\section{Introduction}

Acute respiratory distress syndrome (ARDS) is a life-threatening condition characterized by severe hypoxemia due to respiratory failure that was first described as the "shock lung" by military clinicians in Vietnam in the 1960s [1]. This heterogeneous pathology is now understood to be the cause of death in nearly 200,000 people in the USA each year [2]. While there are many complications that results from ARDS, the development of an acute kidney injury (AKI) is common and can be fatal in these patients. Over the past decade, significant ad-

Manuscript submitted August 7, 2019, accepted October 22, 2019

aDepartment of Medicine, Jersey Shore University Medical Center, Hackensack Meridian Health, Neptune, NJ 07753, USA

bivision of Nephrology, Department of Medicine, Columbia VA Health Care Center, 6439 Garners Ferry Rd, Columbia, SC 29209, USA

'Department of Nephrology and Hypertension, Glickman Urological and Kidney Institute, Cleveland Clinic, Cleveland, OH 44195, USA

${ }^{\mathrm{d}}$ Corresponding Author: Arif Asif, Department of Medicine, Jersey Shore University Medical Center, Hackensack-Meridian School of Medicine at Seton Hall, 1945 Route 33, Neptune, NJ 07753, USA.

Email: arif.asif@hackensackmeridian.org

doi: https://doi.org/10.14740/jocmr3938 vances have been made to understand the pathophysiology of ARDS and complications such as AKI. This article discusses the management of AKI in the setting of ARDS, and highlights its epidemiology, pathophysiology and treatment strategies for internists and intensivists alike.

\section{Definitions}

Acute lung injury (ALI) has been defined as acute lung disease that consists of acute hypoxemic respiratory failure, specifically with ratio of arterial oxygen tension to fraction of inspired oxygen $\left(\mathrm{PaO}_{2} / \mathrm{FiO}_{2}\right)$ of less than $300 \mathrm{~mm} \mathrm{Hg}$ with bilateral pulmonary infiltrates due to pulmonary and non-pulmonary risk factors, and which is not due to left atrial hypertension as per the American/European Consensus Conference definition $[2,3]$. This definition created a distinction between ALI and ARDS based on $\mathrm{PaO}_{2} / \mathrm{FiO}_{2}$ ratio with ARDS categorized as being $<200 \mathrm{~mm} \mathrm{Hg}$ and thus more severe. ARDS was redefined in 2012 and clarifies several uncertain areas not explained by the American/European Consensus conference definition [4]. As per the Berlin definition, ARDS can be diagnosed once cardiogenic pulmonary edema and alternative causes of acute hypoxemic respiratory failure and bilateral infiltrates have been excluded [5]. The Berlin definition of ARDS requires that for the diagnosis to be present all the criteria listed in Table 1 [5] must be present.

For the sake of clarity, this review uses ALI and ARDS interchangeably as to include all patients with a $\mathrm{PaO}_{2} / \mathrm{FiO}_{2}$ less than $300 \mathrm{~mm} \mathrm{Hg}$. In our discussion of AKI, Kidney Disease: Improving Global Outcomes (KDIGO) recommendations for classification of AKI, AKI is defined as an increase in serum creatinine by $\geq 0.3 \mathrm{mg} / \mathrm{dL}$ within $48 \mathrm{~h}$, or an increase of serum creatinine to $\geq 1.5$ times the baseline, which is known to have occurred in the patient within the last 7 days, or a decrease in urine volume of $<0.5 \mathrm{~mL} / \mathrm{kg} / \mathrm{h}$ for $6 \mathrm{~h} \mathrm{[4].} \mathrm{The} \mathrm{literature}$ we review here includes articles published after 2012 and uses KDIGO recommendations for classification for AKI.

\section{Epidemiology}

ARDS has a huge impact on mortality. The Kings County Lung Injury Project (KCLIP) studied the incidence and out- 
Table 1. Diagnostic Criteria for Diagnosis of ARDS (Adapted From the Berlin Definition [5])

Timing: Respiratory symptoms must have an onset within 1 week of known primary insult.

Chest imaging: Includes bilateral opacities not fully explained by effusions, lobar collapse, lung collapse, or nodules on chest X-ray or computed tomographic scan.

Cause of edema: Not fully explained by cardiac cause or fluid overload states with evidence from objective assessment and diagnostic tools required (i.e., echocardiography).

Severity assessment of hypoxemia using ratio of arterial oxygen tension to fraction of inspired oxygen:

Mild: $\mathrm{PaO}_{2} / \mathrm{FiO}_{2}>200 \mathrm{~mm} \mathrm{Hg}$ but $\leq 300 \mathrm{~mm} \mathrm{Hg}$ with PEEP or CPAP $\geq 5 \mathrm{~cm} \mathrm{H}_{2} \mathrm{O}$

Moderate: $\mathrm{PaO}_{2} / \mathrm{FiO}_{2}>100 \mathrm{~mm} \mathrm{Hg}$ but $\leq 200 \mathrm{~mm} \mathrm{Hg}$ with PEEP or CPAP $\geq 5 \mathrm{~cm} \mathrm{H}_{2} \mathrm{O}$

Severe: $\mathrm{PaO}_{2} / \mathrm{FiO}_{2} \leq 100 \mathrm{~mm} \mathrm{Hg}$ with PEEP or CPAP $\geq 5 \mathrm{cmH}_{2} \mathrm{O}$

comes of ALI and had emphasized 75,000 deaths from ALI in the USA each year [6]. These data are comparable to deaths from human immunodeficiency virus (HIV) and breast cancer [6]. Studies have also shown that patients with ARDS who develop AKI demonstrate increased all-cause mortality. Cooke et al found that the relative risk of death from ARDS in patients with oliguric renal failure was 1.97 , where oliguric renal failure was defined as production of $\leq 500 \mathrm{~mL}$ of urine in a $24-\mathrm{h}$ period and a serum creatinine of $\geq 2.0$ on the day of onset [6]. As seen in the ARDSNet trial, patients with AKI and ARDS had close to 2 times the mortality of that seen with ARDS alone (58\% among subjects with AKI compared to $28 \%$ without AKI $(\mathrm{P}<0.001))$ [7]. Newly theorized risk stratified scoring systems have found patients with severe ARDS with hypertension, elevated aspartate aminotransferase levels, and elevated D-dimers had higher risk of developing AKI, and higher risk of mortality [8]. Unfortunately, to gauge a proper understanding of epidemiological trend across multiple studies is difficult as there are multiple barometers of AKI utilized that differ from KDIGO recommendations.

\section{Pathophysiology}

The exact underlying mechanism for AKI in patients with ARDS is an area of intense research, because it is not yet clearly understood. Previous studies have demonstrated that AKI in the setting of ARDS tremendously increases mortality [9]. Adult studies in patients with ARDS have found that $35 \%$ patients end up developing AKI [10]. In one study by Darmon et al, the mortality in patients who have ARDS and AKI was $42.3 \%$, while that in patients without AKI was $20 \%$ [11].

There have been some studies that attempt to explain the pathophysiology of AKI in ARDS. The three main possible mechanisms are mechanical ventilation, hypoxemia and systemic inflammation [11]. However, among these three factors, mechanical ventilation appears to be the most significant event. Mechanical ventilation leads to a cascade of events in multiple organs, including kidney, which eventually leads to AKI thus increasing mortality. There have also been multiple studies that showed that mechanical ventilation can independently cause AKI [12-14].

In one study in a pediatric population, multiple aspects of mechanical ventilation were evaluated as potentially increas- ing risk for AKI in ARDS: peak inspiratory pressure (PIP), positive end expiratory pressure (PEEP), mean airway pressure (MAP) and tidal volume (TV). However, only PEEP was independently associated when adjusted for potential confounders. This association has been reported in multiple previous studies as well [15]. A systematic review and meta-analysis by van Der Akker and his team showed the mechanical ventilation in patients with ARDS increases the risk of developing AKI by almost 3 times. But this analysis failed to identify association of PEEP or TV with AKI [14]. Evidence also indicated that barotrauma caused by high pressure ventilation, not only has potential to injure the lung, but also causes systemic inflammation and organ dysfunction due to release of inflammatory cytokines. Several cytokines such as tumor necrosis factor- $\alpha$ (TNF- $\alpha$ ), transforming growth factor- $\beta 1$ (TGF- $\beta 1$ ), interleukin-1B (IL-1B), IL-6, and IL-8 have been identified with higher rates of $\mathrm{AKI}[7,16,17]$.

In an experimental lung model, Imai and his team found that high alveolar pressures, which occur in the setting of high PEEP, increase incidence of programmed cell death in renal tubules, which might lead to AKI [18]. The consequence of these elevated pressure alter the hemodynamic of the heart, which in turn affects the homeostasis of the kidney. Positive pressure ventilation decreases venous return to the heart, which alters the cardiac preload, pulmonary vascular resistance and afterload to the right side of the heart. All these hemodynamic changes eventually lead to decreased perfusion to all the organs in the body, especially kidneys, leading to reduced glomerular filtration rate (GFR) and AKI [19].

\section{Ventilation Strategies of ARDS and AKI}

Breakthrough studies have shown the benefits of low TV ventilation strategies in the treatment of ARDS. ARDSNet trials performed in the 2000s pivoted the change in the ventilation management of patients in ARDS [20]. Low TV ventilation, described as $6 \mathrm{~mL} / \mathrm{kg}$ TV based on ideal body weight, decreased mortality, duration of intensive care unit (ICU) days, duration of ventilation, and incidence of non-pulmonary organ injury [20]. In one of the studies, non-pulmonary organ injury defined renal failure as serum creatinine concentration of at least $2 \mathrm{mg} / \mathrm{dL}$. Onset of renal failure took longer in low TV ventilation strategy when compared to traditional TV ventila- 
tion $(\mathrm{P}=0.005)[20]$. Benefits of low TV ventilation strategy are seen with mild hypercarbia which can be renal protective but can be complicated with exacerbation of severe metabolic acidosis leading to hemodynamic instability [21]. Studies have demonstrated that higher TVs were associated with increased levels of TNF- $\alpha$, IL-1B, IL-6, and IL-8, with higher rates of AKI or higher number of days with AKI [22]. Moreover, animal models have shown that exposure to moderate hypercapnia $\left(\mathrm{PaCO}_{2} 80\right.$ - 100) is more favorable with less inflammatory injury than animals with severe hypercapnia $\left(\mathrm{PaCO}_{2}\right.$ of $130-$ 150) due to the inhibition of NF-kB expression during increasing levels of hypercapnia [23].

There is still mounting evidence to suggest that increasing deleterious effects of lung dysfunction, its treatment strategies, and subsequent kidney injury explains the natural progression of multi-organ failure seen in critically ill patients. Elevated PEEP, well documented to be beneficial in ARDS management, can also alter hemodynamic changes as it relates to venous return, cardiac afterload, thus decreasing cardiac output along with renal blood flow [15]. Cardiorenal interactions studied by Annat et al has shown that increasing PEEP (PEEP of greater than $10 \mathrm{~cm} \mathrm{H}_{2} \mathrm{O}$ ) leads to significant reductions in urinary output, renal blood flow, sodium excretion, and potassium excretion [21]. These changes were reversed when PEEP was withdrawn. In contrast, studies have also shown ventilation using higher volume and low levels of PEEP lead to increase epithelial injury in nephrons due to a correlation between Fas ligand changes and serum creatinine changes [18]. A large meta-analysis performed in 2013 exhibited a 3 -fold increase in the risk of AKI in both ARDS and mechanical ventilation $[24,25]$. Data have shown independent associations between ARDS and mechanical ventilation to AKI [24]. Thus these studies have proposed that ARDS and mechanical ventilation be listed as risk factors of AKI. A secondary analysis of a multi-center observational study has shown that the presence of AKI was associated with prolonged duration of mechanical ventilation [25]. Patients who received higher TVs on day 1 to 3 upon intubation for ARDS had significantly higher risk of renal nonrecovery [26]. Comparatively, use of lower TVs over the first 3 days of patients diagnosed with ARDS was significantly associated with renal recovery [26]. Long-term consequences of ventilation strategies in the treatment of ARDS to renal physiology remain to be determined [11].

\section{Fluid Management in ARDS and Renal Perfusion}

Often patients with ARDS are given liberal amounts of fluids for management of the underlying cause of systemic injury (e.g. sepsis, trauma, other organ pathology). Initial phases of these conditions necessitate large volume resuscitation in order to achieve and maintain hemodynamic stability [24]. After the return of hemodynamic stability, administration of additional volume has been shown to be detrimental [27]. Inability to preserve lower fluid balance after hemodynamic stability was found to be an independent mortality risk factor in patients who developed ALI in the setting of septic shock
[27]. Compared to the use of liberal fluid strategies, conservative use of normal saline by achieving a negative fluid balance improved oxygenation index, lung injury as well as decreased length of stay in the ICU [28]. Increased renal perfusion with liberal fluid management did not offset the worsening of lung function. There was also less reported use of dialysis in the first 60 days with the conservative fluid strategies. This trial, also known as Fluid and Catheter Treatment Trial (FACTT) study, also showed that administration of intravenous (IV) fluids to shock-free patients did not lead to improvement of kidney function, but only lead to delay in the resolution of lung injury [28]. Overall, the results of this study favored the use of a conservative fluid strategy for critically ill patients with ARDS.

\section{Continuous renal replacement therapy (CRRT) in ARDS}

CRRT has been used as a modality for renal support in critically ill patients in the ICU setting [29]. Continuous venovenous hemofiltration $(\mathrm{CVVH})$ is a CRRT that combines clearance, diffusion, and convection, allowing for the extraction of fluids and electrolytes from a patient's blood [30]. This modality has been useful in removing key humoral mediators of systemic inflammatory response by convection and absorption. Studies have shown the existence of therapeutic benefits of CRRT in patients with ARDS [31]. With the compromised alveolarcapillary barrier function in patients with ARDS, CRRT is beneficial for extracorporeal treatment in maintaining goals for conservative fluid strategy [29]. CRRT is also beneficial in eliminating inflammatory mediators that contribute to the pathogenesis of ARDS. As discussed above, TGF- $\beta 1$ is a key mediator for impeding development ARDS in patients [32]. TGF- $\beta 1$ concentrations have been reported to be substantially elevated in lung fluids in patients with ALI and ARDS [33, 34]. Studies have also shown that after initiation of CRRT, TGF- $\beta 1$ concentrations decreased and were associated with clinical improvement of outcomes in patients based on $\mathrm{PaO}_{2} / \mathrm{FiO}_{2}$ ratios [29]. Data support findings which show that decreased TGF- $\beta 1$ concentrations are associated with more ventilator-free days and ICU-free says [35]. Use of CRRT, when initiated early, is associated with a $28 \%$ mortality risk reduction in patients with AKI in the ICU setting [36]. This statistic was not reproduced in the setting of severe ARDS due to influenza A. In a study looking at the outcomes of AKI in patients with severe ARDS due to influenza A, the need for CRRT was associated with increased mortality [26]. In this population, vasopressor use and duration was associated with CRRT utilization and with increased mortality [37].

However, due to the limitations underlying the results of these studies, conclusions for practical use warrants reexamination. There still exists variability in the defining ARDS in subset of studies as many still use the 1994 AmericanEuropean consensus definition for ARDS, as opposed to the most current Berlin ARDS definition [29]. Several inflammatory markers are involved in the development of ARDS in patients, not all of which have been examined with CRRT. Overall, multifactorial benefit with the use of CRRT for the 
treatment of AKI in the setting of ARDS does merit discussion.

\section{Conclusions}

AKI in conjunction with ARDS increases mortality drastically. Modalities used to treat ARDS also have a pronounced effect in causing kidney injury. This review aims to provide a concise understanding on the intricacies of managing AKI in the setting of primary ARDS as it relates to low TV ventilation and fluid restriction strategies for internists and intensivists alike. Future studies that focus on the cause of death in patients with AKI with ARDS may improve the overall care in this critically ill population.

\section{Acknowledgments}

We take this opportunity to show our gratitude to Dr. Arif Asif in inspiring us and guiding us through the process of writing this manuscript. We are also grateful to Dr. Roy Mathew and Dr. Tushar Vachharajani for their comments and insight into making this manuscript possible. Finally, we are deeply thankful to have had the close support of Dr. Mohammad Hossain, without whom none of this would be possible.

\section{Financial Disclosure}

This project was not supported by any grant or funding agencies.

\section{Conflict of Interest}

The authors have no conflict to disclose.

\section{Author Contributions}

VU and MS conceived the presented idea and wrote the initial draft with the support from AA, MH, RM, and TV, whom were involved in literature review, critical analysis, and in reviewing and editing the final manuscript.

\section{References}

1. Ashbaugh DG, Bigelow DB, Petty TL, Levine BE. Acute respiratory distress in adults. Lancet. 1967;2(7511):319323.

2. Rubenfeld GD, Caldwell E, Peabody E, Weaver J, Martin DP, Neff M, Stern EJ, et al. Incidence and outcomes of acute lung injury. N Engl J Med. 2005;353(16):16851693.

3. Bernard GR, Artigas A, Brigham KL, Carlet J, Falke K, Hudson L, Lamy M, et al. The American-European Con- sensus Conference on ARDS. Definitions, mechanisms, relevant outcomes, and clinical trial coordination. Am J Respir Crit Care Med. 1994;149(3 Pt 1):818-824.

4. Kidney Disease: Improving Global Outcomes (KDIGO) Acute Kidney Injury Work Group. KDIGO clinical practice guideline for acute kidney injury. Kidney Int Suppl. 2012;2:1-138.

5. Force ADT, Ranieri VM, Rubenfeld GD, Thompson BT, Ferguson ND, Caldwell E, Fan E, et al. Acute respiratory distress syndrome: the Berlin Definition. JAMA. 2012;307(23):2526-2533.

6. Cooke CR, Kahn JM, Caldwell E, Okamoto VN, Heckbert SR, Hudson LD, Rubenfeld GD. Predictors of hospital mortality in a population-based cohort of patients with acute lung injury. Crit Care Med. 2008;36(5):1412-1420.

7. Liu KD, Glidden DV, Eisner MD, Parsons PE, Ware LB, Wheeler A, Korpak A, et al. Predictive and pathogenetic value of plasma biomarkers for acute kidney injury in patients with acute lung injury. Crit Care Med. 2007;35(12):2755-2761.

8. Han H, Li J, Chen D, Zhang F, Wan X, Cao C. A clinical risk scoring system of acute respiratory distress syndrome-induced acute kidney injury. Med Sci Monit. 2019;25:5606-5612.

9. Vieira JM, Jr., Castro I, Curvello-Neto A, Demarzo S, Caruso P, Pastore L, Jr., Imanishe $\mathrm{MH}$, et al. Effect of acute kidney injury on weaning from mechanical ventilation in critically ill patients. Crit Care Med. 2007;35(1):184-191.

10. Villacres SM, Medar SS, Aydin SI. Acute kidney injury in children with acute respiratory failure. Clin Pediatr (Phila). 2018;57(11):1340-1348.

11. Darmon M, Clec'h C, Adrie C, Argaud L, Allaouchiche B, Azoulay E, Bouadma L, et al. Acute respiratory distress syndrome and risk of AKI among critically ill patients. Clin J Am Soc Nephrol. 2014;9(8):1347-1353.

12. Chang JW, Jeng MJ, Yang LY, Chen TJ, Chiang SC, Soong WJ, Wu KG, et al. The epidemiology and prognostic factors of mortality in critically ill children with acute kidney injury in Taiwan. Kidney Int. 2015;87(3):632-639.

13. Krishnamurthy S, Narayanan P, Prabha S, Mondal N, Mahadevan S, Biswal N, Srinivasan S. Clinical profile of acute kidney injury in a pediatric intensive care unit from Southern India: A prospective observational study. Indian J Crit Care Med. 2013;17(4):207-213.

14. van den Akker JP, Egal M, Groeneveld AB. Invasive mechanical ventilation as a risk factor for acute kidney injury in the critically ill: a systematic review and metaanalysis. Crit Care. 2013;17(3):R98.

15. Jacob LP, Chazalet JJ, Payen DM, Villiers SM, Boudaoud S, Teillac P, Pruna AS, et al. Renal hemodynamic and functional effect of PEEP ventilation in human renal transplantations. Am J Respir Crit Care Med. 1995;152(1):103-107.

16. Ranieri VM, Giunta F, Suter PM, Slutsky AS. Mechanical ventilation as a mediator of multisystem organ failure in acute respiratory distress syndrome. JAMA. 2000;284(1):43-44.

17. Ranieri VM, Suter PM, Tortorella C, De Tullio R, Dayer JM, Brienza A, Bruno F, et al. Effect of mechanical ven- 
tilation on inflammatory mediators in patients with acute respiratory distress syndrome: a randomized controlled trial. JAMA. 1999;282(1):54-61.

18. Imai Y, Parodo J, Kajikawa O, de Perrot M, Fischer S, Edwards V, Cutz E, et al. Injurious mechanical ventilation and end-organ epithelial cell apoptosis and organ dysfunction in an experimental model of acute respiratory distress syndrome. JAMA. 2003;289(16):2104-2112.

19. de Abreu KL, da Silva Junior GB, Muniz TD, Barreto AG, Lima RS, Holanda MA, Pereira ED, et al. Acute kidney injury in critically ill patients with lung disease: kidneylung crosstalk. Rev Bras Ter Intensiva. 2013;25(2):130136.

20. Acute Respiratory Distress Syndrome N, Brower RG, Matthay MA, Morris A, Schoenfeld D, Thompson BT, Wheeler A. Ventilation with lower tidal volumes as compared with traditional tidal volumes for acute lung injury and the acute respiratory distress syndrome. N Engl J Med. 2000;342(18):1301-1308.

21. Annat G, Viale JP, Bui Xuan B, Hadj Aissa O, Benzoni D, Vincent M, Gharib C, et al. Effect of PEEP ventilation on renal function, plasma renin, aldosterone, neurophysins and urinary $\mathrm{ADH}$, and prostaglandins. Anesthesiology. 1983;58(2):136-141.

22. Andres-Hernando A, Dursun B, Altmann C, Ahuja N, He Z, Bhargava R, Edelstein CE, et al. Cytokine production increases and cytokine clearance decreases in mice with bilateral nephrectomy. Nephrol Dial Transplant. 2012;27(12):4339-4347.

23. Yang W, Yue Z, Cui X, Guo Y, Zhang L, Zhou H, Li W. Comparison of the effects of moderate and severe hypercapnic acidosis on ventilation-induced lung injury. BMC Anesthesiol. 2015;15:67.

24. Rivers E, Nguyen B, Havstad S, Ressler J, Muzzin A, Knoblich B, Peterson E, et al. Early goal-directed therapy in the treatment of severe sepsis and septic shock. N Engl J Med. 2001;345(19):1368-1377.

25. McNicholas BA, Rezoagli E, Pham T, Madotto F, Guiard E, Fanelli V, Bellani G, et al. Impact of early acute kidney injury on management and outcome in patients with acute respiratory distress syndrome: a secondary analysis of a multicenter observational study. Crit Care Med. 2019;47(9):1216-1225.

26. Panitchote A, Mehkri O, Hastings A, Hanane T, Demirjian S, Torbic H, Mireles-Cabodevila E, et al. Clinical predictors of renal non-recovery in acute respiratory distress syndrome. BMC Nephrol. 2019;20(1):255.

27. Cerda J, Sheinfeld G, Ronco C. Fluid overload in critically ill patients with acute kidney injury. Blood Purif. 2010;29(4):331-338.

28. Wiedemann HP, Wheeler AP, Bernard GR, Thompson BT, Hayden D, et al. Comparison of two fluid-management strategies in acute lung injury. N Engl J Med. 2006;354(24):2564-2575.

29. Han F, Sun R, Ni Y, Hu X, Chen X, Jiang L, Wu A, et al. Early initiation of continuous renal replacement therapy improves clinical outcomes in patients with acute respiratory distress syndrome. Am J Med Sci. 2015;349(3):199205.

30. Blankestijn PJ, Ledebo I, Canaud B. Hemodiafiltration: clinical evidence and remaining questions. Kidney Int. 2010;77(7):581-587.

31. Saito A. Current progress in blood purification methods used in critical care medicine. Contrib Nephrol. 2010;166:100-111.

32. de Pablo R, Monserrat J, Reyes E, Diaz D, RodriguezZapata M, la Hera A, Prieto A, et al. Sepsis-induced acute respiratory distress syndrome with fatal outcome is associated to increased serum transforming growth factor beta-1 levels. Eur J Intern Med. 2012;23(4):358-362.

33. Budinger GR, Chandel NS, Donnelly HK, Eisenbart J, Oberoi M, Jain M. Active transforming growth factor-beta1 activates the procollagen I promoter in patients with acute lung injury. Intensive Care Med. 2005;31(1):121128.

34. Fahy RJ, Lichtenberger F, McKeegan CB, Nuovo GJ, Marsh CB, Wewers MD. The acute respiratory distress syndrome: a role for transforming growth factor-beta 1 . Am J Respir Cell Mol Biol. 2003;28(4):499-503.

35. Dhainaut JF, Charpentier J, Chiche JD. Transforming growth factor-beta: a mediator of cell regulation in acute respiratory distress syndrome. Crit Care Med. 2003;31(4 Suppl):S258-264.

36. Seabra VF, Balk EM, Liangos O, Sosa MA, Cendoroglo $\mathrm{M}$, Jaber BL. Timing of renal replacement therapy initiation in acute renal failure: a meta-analysis. Am J Kidney Dis. 2008;52(2):272-284.

37. Tignanelli CJ, Wiktor AJ, Vatsaas CJ, Sachdev G, Heung M, Park PK, Raghavendran K, et al. Outcomes of acute kidney injury in patients with severe ARDS due to influenza A(H1N1) pdm09 virus. Am J Crit Care. 2018;27(1):67-73. 